Case Report

\title{
Re-igniting the PE debate
}

\author{
Avinash Aujayeb ${ }^{1 *}$, Joseph Mackenzie ${ }^{2}$ \\ ${ }^{1 *}$ Consultant in Respiratory and General Medicine, North Tyneside General Hospital, Northumbria Health Care Foundation Trust, UK. \\ ${ }^{2}$ Specialist Registrar in Respiratory Medicine, North Tyneside General Hospital, Northumbria Health Care Foundation Trust, UK.
}

*Correspondence to: Avinash Aujayeb, Consultant in Respiratory and General Medicine, North Tyneside General Hospital, Northumbria Health Care Foundation Trust, UK; E-mail: aujayeb@doctors.net.uk

Received: October 25, 2016; Accepted: November 04, 2016; Published: November 11, 2016

\begin{abstract}
We describe the case of patient with a sub-massive pulmonary embolus and the evidence based management.

Learning points: The decision to thrombolyse or not to thrombolyse a sub-massive PE is difficult and several clinical factors need to be taken into consideration
\end{abstract}

Key words: sub-massive, pulmonary embolus, thrombolysis

Joseph Mackenzie (JM): A 78 year old female patient presented 4 days ago with 3 day history of sudden onset breathlessness and dizziness. She was known to have chronic unspecified gromerulonephritis and had a WHO performance score of 1 . Her regular medications included anti-hypertensives and quinine for night cramps. She was a never smoker with no dust exposure.

Initially, her pulse rate was 76 beats per minute, her oxygen saturations 95 per cent on air and blood pressure 105 over $76 \mathrm{~mm}$ of mercury. Her inflammatory markers were normal but her urea and creatinine had risen to $27.9 \mathrm{mmol}$ per litre and $238 \mathrm{ummol}$ per litre. Her chest radiograph and electrocardiogram was normal.

She was admitted under the care of the elderly team, was rehydrated and her nephrotoxics stopped. On the second day of admission, she had a syncopal episode with a spontaneous return to circulation: An arterial blood gas done on air showed a $\mathrm{pCO}^{2}$ of $3.2 \mathrm{KPascals}$, a $\mathrm{pO}^{2}$ of $8.9 \mathrm{KPascals}$, a base excess of $-10 \mathrm{mmol} / \mathrm{L}$ and oxygen saturations of 94 per cent.

The next day, a D-dimer was checked- that was more than 20.00 $\mathrm{mg} / \mathrm{L}$ (normal being $<0.50$ ). Her hypotension was fluctuating. An echocardiogram was done- it showed a flattened septum throughout the cardiac cycle suggesting right ventricular (RV) overload, mildly impaired systolic function, moderate tricuspid regurgitation with a pulmonary arterial systolic pressure (PASP) estimated at $70 \mathrm{~mm}$ mercury added to the right atrial pressure and a tricuspid annular plane systolic excursion of $1 \mathrm{~cm}$.

A computed tomography pulmonary angiogram was then performed which showed massive pulmonary embolism(PE) bilaterally in upper, middle and lower lobe pulmonary arteries, bilateral main pulmonary arteries (Fig 1) with significant right ventricular strain noted with reflux of contrast into IVC and hepatic veins.

She was started on full dose low molecular weight heparin and warfarin. Her current International Normalised Ratio (INR) is 1.8
Avinash Aujayeb (AA): Her initial Wells score was $3^{1}$ (only scored yes for PE being likely diagnosis), which puts her in a moderate risk group and combined with a high D dimer initially, she certainly warranted further investigation.

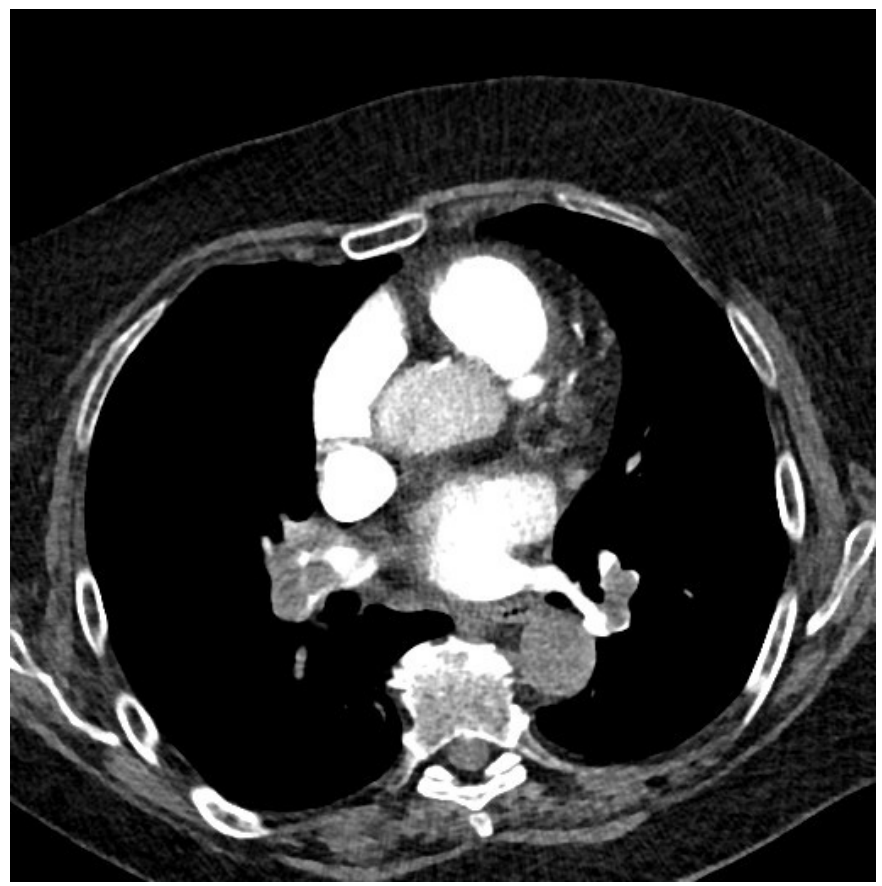

Figure 1. CT scan showing massive bilateral pulmonary emboli and RV strain

Her alveolar-arterial oxygen tension difference $\left(P_{\mathrm{A}-\mathrm{aO} 2}\right)$ was $7 \mathrm{kPa}$.

The $P_{\mathrm{A}-\mathrm{aO} 2}$ is a measure of the difference between the alveolar concentration (A) of oxygen and the arterial (a) concentration of oxygen. In room air (inspiratory oxygen fraction of 0.21 ) at sea level (atmospheric pressure of $760 \mathrm{mmHg}$ ) assuming 100\% humidity in the alveoli, a simplified version of the equation is $21-\left(\left(P_{\mathrm{aCO} 2} / 0.8\right)-P_{\mathrm{aO} 2}\right)$, 
0.8 being the respiratory quotient. Whilst her peripheral saturations might appear normal, her high tension difference suggests a ventilation-perfusion mismatch and in a never smoker with a normal chest radiograph, a vascular event needs excluding.

The echocardiographic findings are worrisome. The main cause of death in acute PE is RV failure due to pressure overload. The abrupt increase in pulmonary vascular resistance results in RV dilation, which alters the contractile properties of the RV myocardium via the Frank-Starling mechanism. The increase in the RV afterload also causes the tricuspid valve to fail and the PASP is a marker of that. TAPSE is a simple echocardiographic measure of RV ejection fraction and any value below $2 \mathrm{~cm}$ is considered normal. Echocardiographic examination is not recommended as part of the diagnostic work-up in haemodynamically stable, normotensive patients with suspected (not high-risk) $\mathrm{PE}^{2}$, but in a suspected high-risk $\mathrm{PE}$, a normal echocardiogram can exclude it.

Joseph Mackenzie (JM): The patient has had no further syncopal episodes but has been more breathless today, and is now requiring 2 litres via nasal cannulae to maintain oxygen saturations at $95 \%$. Her respiratory rate has increased to 26 per minute and her blood pressure chart is as in Figure 2. A troponin $\mathrm{T}$ is 114 nanograms/L (normal range $0-14$ ) and her lactate is $3.3 \mathrm{millimol} / \mathrm{L}$ (normal $0.5-2.2$ )- that was previously 1.9. This is now almost 7 days after she developed the initial symptoms. I note her INR of 1.8 but wonder if she would benefit from thrombolysis.

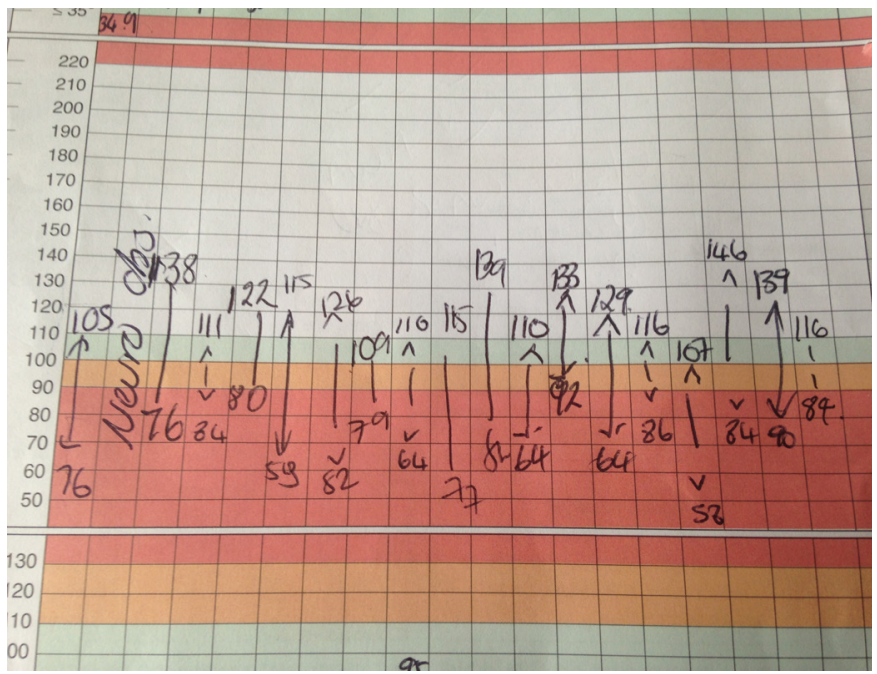

Figure 2. Observation chart showing fluctuations in blood pressure

Avinash Aujayeb (AA): Patients with PE and shock or hypotension are at high risk of death, particularly in the first few hours after admission. The clinical classification an acute PE is based on estimated early mortality risk (in-hospital or 30 -day) ${ }^{2}$ and shock or hypotension in $\mathrm{PE}$ is defined as a systolic blood pressure less than $90 \mathrm{~mm}$ of mercury or a systolic sustained drop of more than $40 \mathrm{~mm}$ of mercury over more than 15 minutes, in the absence of arrythmia, hypovolemia and sepsis. Hence, by strict definition, at the moment, she has a submassive PE (confirmed PE in a normotensive patient with evidence of RV dilatation and/or RV dysfunction and/or pulmonary hypertension) but I note the fluctuation hypotension.

There have been excellent debates of the pros and cons of thrombolysis in submassive PE recently.

The con arguments $^{3}$ are that large registries suggest 90 day mortality in thrombolysed patients to be around $3 \%$ and in the heparin only group to be around $2 \%$, that RV dilatation is a dynamic process and some studies have shown that $93 \%$ of such patients have normalised their RV at 6 months and that there is no evidence proving that early haemodynamic improvements has survival benefits, prevents recurrence and development of chronic thromboembolic pulmonary hypertension. The PETHIO trials ${ }^{2}$ also suggested statistically significant differences in bleeding complications (2\% incidence of haemorrhagic stroke after thrombolytic treatment and $6 \%$ risk of major non-intracranial bleeding events).

However, I think that her initial presenting symptoms are important and I agree with thrombolysis. There is a relative contraindication that her INR is 1.8 but I think her risk of death is high.

I have sought a second opinion on this from a cardiologist who agrees with thrombolysis.

She has markers of significant myocardial necrosis and RV dysfunction. Her initial presentation with syncope and hypotension is an adverse prognostic sign and even though her outward haemodynamics have normalised now, her rising lactate suggests otherwise. Thrombolysis can quickly restore pulmonary perfusion and resolve pulmonary resistance to improve RV function ${ }^{4}$. The greatest benefit occurs when the agent is administered within 48 hours of the primary event, but benefit has been proven for patients up to 14 days down the line ${ }^{2}$.

The ESC guidelines ${ }^{2}$ would classify her to be in the intermediate high risk group, where thrombolysis should not be routinely considered, unless there is haemodymanic decompensation. A rising lactate is a proven maker of this ${ }^{5}$.

Joseph Mackenzie (JM): The patient provided written consent to thrombolysis.

$10 \mathrm{mg}$ of alteplase was given over 2 minutes followed by $90 \mathrm{mg}$ over 2 hours. Continuous haemodynamic and neurological monitoring was performed with no anomalies detected. There was a small brisk episode of epistaxis which was self limiting and subcutaneous bruising appeared. Within 4 hours of administration, oxygen levels increased to 100 per cent on $2 \mathrm{~L}$ nasal cannulae and remained at $96 \%$ on air afterwards. The patient's breathlessness disappeared and full dose low molecular weight heparin was restarted as well as warfarin loading.

After a brief period of rehabilitation, she has now been discharged and will have follow up in the thrombosis clinic with a repeat echocardiogram in 3 months. 


\section{References}

1. http://www.mdcalc.com/wells-criteria-for-pulmonary-embolism-pe/ (Accessed 3.3.16)

2. http://www.escardio.org/Guidelines-\&-Education/Clinical-Practice-Guidelines/ Acute-Pulmonary-Embolism-Diagnosis-and-Management-of (Accessed 3.3.16)

3. Simpson AJ (2014) Thrombolysis for acute submassive pulmonary embolism: CON viewpoint. Thorax 69: 105-107. [crossref]

4. Howard LS (2014) Thrombolytic therapy for submassive pulmonary embolus? PRO viewpoint Thorax 69:103-5.

5. Fuller, Brian M, Phillip Dellinger R (2012) "Lactate as a Hemodynamic Marker in the Critically Ill." Current opinion in critical care 18.3 267-272. 